\title{
The Wide-Awake Approach to Dupuytren's Disease: Fasciectomy under Local Anesthetic with Epinephrine
}

\author{
Rebecca Nelson • Amanda Higgins • Joanie Conrad • \\ Mike Bell • Don Lalonde
}

Received: 5 July 2009 /Accepted: 13 October 2009/Published online: 10 November 2009

(C) American Association for Hand Surgery 2009

\begin{abstract}
The Wide-Awake Approach to Dupuytren's contracture involves fasciectomy under local anesthetic with epinephrine and no tourniquet. The goal of this study is to show that the Wide-Awake Approach produces equivalent outcomes to fasciectomy under general anesthetic with a tourniquet, with fewer risks to the patient. A multicenter retrospective review was conducted on 111 patients with fasciectomies under local or general anesthetic between 2001 and 2007. Data on patient demographics, comorbidities, cost, as well as range of motion was collected and evaluated using Microsoft Excel and SAS. Of 148 fingers, 102 were treated under local and 46 under general anesthetic. The average
\end{abstract}

This paper was presented at the American Society for Surgery of the Hand Annual Meeting, Seattle, WA (September 2007) and at the Canadian Society for Plastic Surgery Annual Meeting, St. John's, NFLD, Canada (June 2008). It received the J. F. Murray Award for best paper in hand surgery at the Canadian Society for Plastic Surgery Meeting in June 2008.

R. Nelson $(\bowtie) \cdot$ D. Lalonde

Department of Plastic Surgery, Dalhousie University,

Suite C204, 600 Main St,

Saint John, NB E2K 1J5, Canada

e-mail: r.nelson@dal.ca

\section{A. Higgins}

Department of Occupational Therapy, Dalhousie University,

Suite C204, 600 Main St,

Saint John, NB E2K 1J5, Canada

J. Conrad

Department of Plastic Surgery, University of Ottawa,

1919 Riverside Drive, Suite 402,

Ottawa, ON, Canada K1H 1A2

M. Bell

Department of Occupational Therapy, University of Ottawa,

1919 Riverside Drive, Suite 402,

Ottawa, ON, Canada K1H 1A2 postoperative Total Active Motion (TAM) for general anesthetic patients was $199.0 \pm 29.6$ (D5), $223.9 \pm 29.3$ (D4), $234.6 \pm 14.6$ (D3), and $246.7 \pm 14.4$ (D2). The average postoperative TAM for local anesthetic patients was $168.3 \pm 62.2$ (D5), $195.9 \pm 67.5$ (D4), $173.0 \pm 72.6$ (D3), and $177.5 \pm 31.8$ (D2). There were no significant differences between any of these individual groups ( $p=0.09,0.26,0.12$, and 0.20 , respectively); however, when pooled, the overall TAM was significantly greater in the general anesthesia group (222.0 \pm 29.7 vs. $186.0 \pm 63.0, p=0.002$.). Complication rates and types were similar with both techniques. The Wide-Awake Approach to Dupuytren's contracture avoids general anesthetic risks and has cost benefits to healthcare providers. Although it yields similar range of motion outcomes to fasciectomy performed under general anesthesia, total active motion may be better with fasciectomy done under general anesthesia.

Keywords Anesthesia · Dupuytren's contracture · Epinephrine $\cdot$ Motion

\section{Introduction}

Digital and palmar fasciectomy for Dupuytren's disease is a common procedure performed by plastic surgeons. Historically, surgery has been done in the main operating room under general anesthesia, using a tourniquet. Two main factors have made it difficult for surgeons to perform this surgery under local anesthesia. Both of these are related to maintaining a bloodless field in which to operate. The first is the use of an arm or forearm tourniquet, which are not tolerated for long periods of time by awake patients because of pain and discomfort $[5,16,27]$. The second limitation is the 
avoidance of epinephrine use in the digits, which stems from the previously held belief that epinephrine should be avoided in the finger [11, 20, 29, 34].

The Dalhousie project included 3,110 consecutive cases of hand and finger epinephrine injection without the use of arm tourniquet and has proven the safety of this method in many types of hand surgery [20]. This "Wide-Awake Approach" paper showed that there were no ischemic complications in 203 consecutive cases of Dupuytren's fasciectomy done over the period of 2002-2004 [20]. This technique avoided the use of an arm tourniquet and general anesthesia, completely eliminating adverse effects of general anesthesia such as nausea and vomiting and unwanted admissions to hospital. Other series have also demonstrated similar results $[2-4,12,18,19,31,33,34$, 36 and have shown the safety of digital blocks with local anesthetic with epinephrine at a dose of $\leq 1: 100,000$.

There is no study in the literature to date which compares range of motion data on patients with Dupuytren's fasciectomy done under pure local vs. general anesthesia methods. Similarly, there is minimal data comparing complication rates and costs between these two groups.

The goal of this study was to retrospectively review cases of Dupuytren's disease treated at two Canadian centers under either general or local anesthesia with the Wide-Awake Approach. Using change in extension and Total Active Motion (TAM) data, we sought to determine if the change in range of motion, complication rates, and costs were different for patients having fasciectomy under general vs. local anesthesia with epinephrine and no tourniquet. We hypothesized that there would be no significant difference in postoperative range of motion between the two groups, whereas patients having general anesthesia would have higher complication rates and costs. This is the first study to review the outcomes in these two groups of Dupuytren's patients.

\section{Materials and Methods}

A literature search was initially performed combining the MeSH terms Dupuytren's, local anesthetic, epinephrine, digit, and tourniquet. Prior to commencing the study, ethics approval was obtained at two separate institutions in two provinces, and patients qualifying for the study were retrospectively identified through each surgeon's office records. Hospital chart reviews were performed on 120 patients undergoing Dupuytren's fasciectomies at two institutions from 2001 to 2007. Surgeries were performed by four individual surgeons in two different cities, in either (1) minor surgery clinic or in the main operating room under pure local anesthetic (Wide-Awake Approach) or (2) under general anesthesia. Patients were booked for general anesthesia or local anesthesia as per surgeon preference, with two surgeons using predominantly general anesthesia and two surgeons using predominantly local anesthesia. Patients having general anesthetics were admitted as day surgery patients and discharged home the same day as surgery, unless general anesthesia requirements or complications forced an overnight stay.

Individuals undergoing local anesthesia received between 10 and $20 \mathrm{~cm}^{3}$ of lidocaine $1 \%$ with epinephrine (one in $100,000)$ infiltrated into the affected palm and digit(s), without the use of a tourniquet. They received no sedation. For general anesthetic cases, patients had surgery performed with the use of a tourniquet. In these cases, no local anesthetic was injected into the surgical site preoperatively. All surgery was performed under loupe magnification (3.5× power).

Demographic data was collected on patients, along with operative details and outcomes. Occupational therapy charts were used to obtain range of motion data for joints in the involved digits, and TAM was calculated. The range of motion of each finger joint (MP, PIP, DIP) was measured with a goniometer, and the sum of active flexion minus active extension deficits of these three joints was calculated. Most patients visited a hand therapist preoperatively for baseline measurements, and all patients underwent therapy postoperatively. Data were compared between patients in the local anesthetic with epinephrine and no tourniquet vs. general anesthetic with tourniquet groups to see if there were any differences in surgical complications or mobility outcomes. The costs of these two methods were then estimated and compared to determine any cost savings associated with either method.

Costs were determined through our hospital ordering departments and reflect the costs at only one center. Resources for anesthetists fee include the provincial billing schedule and a staff anesthetist, reflecting a 2-h Dupuytren's case. The drug cost includes basic induction with $100 \mu \mathrm{g}$ Fentanyl (\$0.39), $200 \mathrm{mg}$ Propofol (\$3.20), and maintenance with Sevofluorane $\left(10 \mathrm{~cm}^{3}\right.$ of a $250-\mathrm{cm}^{3}$ bottle, approximately $\left.\$ 7.94\right)$. The cost of antiemetics was not included in the cost estimate, nor was the cost of anesthesia machine maintenance or tourniquet maintenance. Costs associated with LMA use and autoclaving were obtained from our hospital ordering department and compared with previous studies in the literature for accuracy [22]. The cost per LMA reflects $\$ 270$ per new mask, with 40 uses per mask; therefore, cost per use is $270 / 40$ or $\$ 6.75$.

In order to determine the cost savings and impact associated with performing fasciectomies under local anesthesia, we asked attending surgeons at our two institutions to fill out a survey (Fig. 1). The survey inquired about the number of Dupuytren's cases they performed annually and whether they were done under local or general 
Figure 1 Dupuytren's survey.

1. How many Dupuytren's fasciectomies do you perform per year?

2. What percent are performed in the:

(\%) Main OR

(\%) Clinic/Office

3. What percentage of your patients have fasciectomies under:

(\%) General anesthesia

(\%) Regional anesthesia

(\%) Local anesthesia

4. If you perform fasciectomy under local anesthesia, do you operate on:

$$
\begin{aligned}
& \text { single digit disease } \pm \text { palmar disease only? } \quad \mathrm{Y} / \mathrm{N} \\
& \text { both single and mulit-digit disease } \pm \text { palmar disease? } \quad \mathrm{Y} / \mathrm{N} \\
& \begin{array}{ll}
\text { recurrent disease? } & \mathrm{Y} / \mathrm{N}
\end{array}
\end{aligned}
$$

3. In what percentage of cases do you use:

$(\%)$ a tourniquet?

(\%) local anesthesia WITHOUT epinephrine?

(\%) local anesthesia WITH epinephrine?

4. What percentage of the patients you operate on have:

(\%) single digit disease \pm disease in the palm

(\%) multi-digit disease \pm disease in the palm

(\%) recurrent disease

5. Do you routinely send patients to OT/PT for hand therapy

preoperatively? Y / N

postoperatively? Y / N

6. Do you routinely use splints:

In the immediate post-operative period?

$\mathrm{Y} / \mathrm{N}$ For how long?

In the late post-operative period (i.e. night splints)? Y / N For how long? 
anesthesia. We also asked about the use of splints and the estimated cost savings if surgery was performed under local instead of general. We received 24 responses from surgeons affiliated with our two institutions, yielding a response rate of $100 \%$.

Statistical analysis was performed using Microsoft Excel Office Professional Edition (Microsoft Corporation, Redmond, WA) 2003 and SAS 9.1 (SAS Institute Inc., Cary, NC) software, using Fisher's exact and Chi square tests for binary data. The Student's $t$ test was used to analyze normally distributed continuous data, and the Wilcoxon rank sum test was used to analyze continuous data with nonparametric distributions. A post hoc power calculation was performed for pooled TAM data, giving this calculation $99 \%$ power.

\section{Results}

Our study was comprised of 111 patients with 148 digital fasciectomies. The general anesthesia group included 46 digits (24 patients), and the local anesthesia group included 102 digits (87 patients). Both groups were predominantly male, with no significant difference between the two (Table $1 ; p=0.20$ ). The average age of individuals having general anesthetics with tourniquets was $65.4 \pm 7.4$ years, compared with $65.5 \pm 11.0$ years in the local anesthesia group with epinephrine. There was no significant difference in age between the two groups $(p=0.63)$. The ring and little fingers were the most frequently involved in both groups, followed by the long and then index finger (Table 1). The thumb was rarely involved in either group. The only significant difference between our two groups was that individuals undergoing general anesthesia had fasciectomies of multiple digits more often at the time of surgery, whereas individuals having local anesthesia had more commonly a single-digit fasciectomy $(p<0.0001)$.

Both groups had more frequent involvement of their right hands $(57.5 \%$ and $54.2 \%$ for local and general groups, respectively; $p=0.77$ ). In addition, both groups had similar percentages of recurrent cases $(2.2 \%$ vs. $4.9 \%$ for local vs. general, respectively; $p=1.00$ ). Complications were similar in both groups, and no significant differences existed with respect to delayed healing, digital nerve lacerations, infection, or other complication $(p=0.75$; Table 1). There was no significant difference between their length of postoperative therapy $(11.5 \pm 7.4$ vs. $8.0 \pm$ 8.6 weeks; $p=0.22$ ).

Preoperative extensor lags for the general anesthesia group are seen in Table 2. There were no significant differences in preop extension for MP or PIP joints for any

Table 1 Demographics.

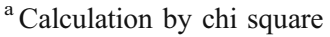

${ }^{\mathrm{b}}$ Calculation by Student's $t$ test

${ }^{\mathrm{c}}$ Calculation by Fischer's exact
}

\begin{tabular}{|c|c|c|c|}
\hline Factor & $\begin{array}{l}\text { General anesthesia with } \\
\text { tourniquet ( } n=46 \text { digits in } \\
24 \text { patients) }\end{array}$ & $\begin{array}{l}\text { Local anesthesia with } \\
\text { epinephrine and no tourniquet } \\
\text { ( } n=102 \text { digits in } 87 \text { patients) }\end{array}$ & $p$ Value \\
\hline \multicolumn{4}{|l|}{ Gender } \\
\hline Male & $18(75.0 \%)$ & $75(85.0 \%)$ & \multirow[t]{2}{*}{$0.20^{\mathrm{a}}$} \\
\hline Female & $6(25.0 \%)$ & $12(15.0 \%)$ & \\
\hline Age & $65.4 \pm 7.4$ & $65.5 \pm 11.0$ & $0.63^{\mathrm{b}}$ \\
\hline \multicolumn{4}{|l|}{ Digits involved } \\
\hline Little & $16(34.8 \%)$ & $48(47.0 \%)$ & $0.16^{\mathrm{a}}$ \\
\hline Ring & $18(39.1 \%)$ & $40(39.2 \%)$ & $0.99^{\mathrm{a}}$ \\
\hline Long & $8(17.4 \%)$ & $10(9.8 \%)$ & $0.19^{\mathrm{a}}$ \\
\hline Index & $3(6.5 \%)$ & $3(2.9 \%)$ & $0.38^{\mathrm{c}}$ \\
\hline Thumb & $1(2.2 \%)$ & $1(1.0 \%)$ & $0.53^{\mathrm{c}}$ \\
\hline Total & 46 & 102 & \\
\hline Single & $11(45.8 \%)$ & $72(82.8 \%)$ & \multirow[t]{2}{*}{$<0.0001^{\circ}$} \\
\hline Two or more & $35(76.1 \%)$ & $30(9.8 \%)$ & \\
\hline Right side & $13(54.2 \%)$ & $50(57.5 \%)$ & $0.77^{\mathrm{a}}$ \\
\hline Recurrent disease & $1(2.2 \%)$ & $5(4.9 \%)$ & $1.00^{\mathrm{c}}$ \\
\hline Length of therapy (weeks) & $11.5 \pm 7.4$ & $8.0 \pm 8.6$ & $0.22^{\mathrm{b}}$ \\
\hline Complications & $3(6.5 \%)$ & $9(8.8 \%)$ & $0.75^{\mathrm{c}}$ \\
\hline Delayed healing & $1(2.2 \%)$ & $3(2.9 \%)$ & $1.00^{\mathrm{c}}$ \\
\hline Nerve laceration & $2(4.3 \%)$ & $2(2.0 \%)$ & $0.59^{\mathrm{c}}$ \\
\hline Infection & 0 & $2(2.0 \%)$ & $1.00^{\mathrm{c}}$ \\
\hline Other & 0 & $2(2.0 \%)$ & $1.00^{\mathrm{c}}$ \\
\hline
\end{tabular}


Table 2 Average preoperative extension.

\begin{tabular}{lccc}
\hline Joint & General anesthesia & Local anesthesia & $p$ Value \\
\hline D5 MP & $-35 \pm 7.1$ & $-49.0 \pm 24.8$ & 0.78 \\
D5 PIP & $-75 \pm 21.2$ & $-62.1 \pm 28.0$ & 0.72 \\
D4 MP & $-40 \pm 0$ & $-44.0 \pm 21.4$ & 0.93 \\
D4 PIP & $-90 \pm 0$ & $-55.8 \pm 29.2$ & 0.61 \\
D3 MP & $-40 \pm 0$ & $-34.0 \pm 15.2$ & 0.67 \\
D3 PIP & $-70 \pm 0$ & $-55.0 \pm 20.2$ & 1.00 \\
\hline
\end{tabular}

$p$ Values calculated by Student's $t$ test

digits recorded (little, ring, long). We did not evaluate range of motion data on the index or thumb because of small numbers ( $n=1$ thumb per anesthesia group and $n=3$ index fingers per group). In addition, we had limited data on DIP joint motion as this joint was generally not involved. Postoperative extension values are seen in Table 3. There were significant differences in average postop extension values for the ring and little fingers ( $p=0.02$ for little finger PIPJ and $p=0.05$ for the ring finger MPJ). This suggested that patients undergoing local anesthetic had better postop extension than those undergoing general anesthetic. There were no other significant differences in postop extension between the two groups.

When analyzing the average change in extension from preoperative to postoperative mobility status, there were no significant differences between local and general anesthesia groups for any digit or joint (Table 4 , all $p$ values $>0.05$ ). Similarly, there was no significant difference in TAM between individual fingers operated on under local vs. general anesthetic (Table 5). When Total Active Motion data were pooled for all digits and compared, the general anesthesia patients had better TAM than patients done under local (Table 5).

A cost comparison was performed by determining the average cost of Dupuytren's fasciectomy at one of our facilities. General anesthesia costs were determined from base drug costs, $2 \mathrm{~h}$ of billing by the anesthetist, OR labor, and materials costs. Clinic costs were determined from a previous study performed at our hospital [21] and reflect

Table 3 Average postoperative extension.

\begin{tabular}{lccc}
\hline Joint & General anesthesia & Local anesthesia & $p$ Value \\
\hline D5 MP & $-5.0 \pm 5.0$ & $6.0 \pm 22.0$ & 0.06 \\
D5 PIP & $-24.0 \pm 17.0$ & $-14.0 \pm 25.0$ & 0.02 \\
D4 MP & $-1.3 \pm 3.2$ & $3.4 \pm 21.0$ & 0.05 \\
D4 PIP & $-11.0 \pm 13.7$ & $-4.0 \pm 20.3$ & 0.09 \\
D3 MP & $-4.1 \pm 5.0$ & $4.0 \pm 12.4$ & 0.23 \\
D3 PIP & $-3.0 \pm 7.1$ & $-13.7 \pm 20.8$ & 1.00 \\
\hline
\end{tabular}

$p$ Values calculated by Student's $t$ test
Table 4 Average change in extension.

\begin{tabular}{llcl}
\hline Joint & General anesthesia & Local anesthesia & $p$ Value \\
\hline D5 MPD3 & $35.0 \pm 7.1$ & $48.5 \pm 31.1$ & 0.92 \\
D5 PIP & $33.0 \pm 9.9$ & $47.9 \pm 26.5$ & 0.56 \\
D5 DIP & na & $23.4 \pm 10.1$ & na \\
D4 MP & $40.0 \pm 0$ & $42.8 \pm 25.9$ & 1.00 \\
D4 PIP & $62.0 \pm 0$ & $44.4 \pm 26.0$ & 0.39 \\
D4 DIP & na & $45.0 \pm 39.0$ & na \\
D3 MP & $28.0 \pm 0$ & $26.4 \pm 17.7$ & 1.00 \\
D3 PIP & $70.0 \pm 0$ & $36.5 \pm 14.7$ & 0.22 \\
D3 DIP & na & $45.0 \pm 21.2$ & na \\
D2 PIP & na & $45.0 \pm 0$ & na \\
D1 MP & na & $25.0 \pm 0$ & na \\
\hline
\end{tabular}

$p$ Values calculated by Student's $t$ test

$n a$ data not available

the costs in one province. As seen in Table 6, we determined that the average cost of performing a fasciectomy in clinic is $\$ 36.46$, while in the main OR under general anesthesia, the cost is $\$ 468.82$. The difference in cost is $\$ 432.36$ Canadian dollars per patient. Our analysis shows that there was a significant cost savings associated with the Wide-Awake Approach when compared with Dupuytren's in the main operating room with a tourniquet and general anesthetic.

To determine the relevance of this cost savings, we polled the attending surgeons at our two institutions in order to determine their current practice profiles. This included some surgeons who perform Dupuytren's under regional block anesthesia, whose patients are not included in this study. The average number of Dupuytren's fasciectomy cases performed per year was $25 \pm 19$ (range 4-85). Of the surgeons polled, $75.0 \%(18 / 24)$ perform the majority $(\geq 50 \%)$ of their Dupuytren's cases main OR. Interestingly, less than half of these patients $(29.5 \%)$ have general anesthetics. The majority has regional anesthesia $(40.8 \%)$, and the remainder have local anesthesia (29.7\%). Tourniquets are used $74.7 \%$ of the time for these cases.

The remaining $20.8 \%(5 / 24)$ of surgeons perform the majority of their Dupuytren's fasciectomies $(\geq 80 \%)$ in the

Table 5 Postoperative TAM.

\begin{tabular}{lccc}
\hline Digit & General anesthesia & Local anesthesia & $p$ Value \\
\hline Little & $199.0 \pm 29.6$ & $168.3 \pm 62.2$ & 0.09 \\
Ring & $223.9 \pm 29.3$ & $195.9 \pm 67.5$ & 0.26 \\
Long & $234.6 \pm 14.6$ & $173.0 \pm 72.6$ & 0.12 \\
Index & $246.7 \pm 14.4$ & $177.5 \pm 31.8$ & 0.20 \\
Overall & $217.0 \pm 31.9$ & $181.2 \pm 65.6$ & 0.0006 \\
\hline
\end{tabular}

$p$ Values calculated by Student's $t$ test 
Table 6 Cost comparison for day surgery under general anesthesia vs. minor surgery in the clinic.

\begin{tabular}{lcc}
\hline Item & $\begin{array}{l}\text { Main OR } \\
\text { cost }(\$)\end{array}$ & $\begin{array}{l}\text { Minor surgery } \\
\text { cost }(\$)\end{array}$ \\
\hline General anesthesia & & \\
Drug costs & 11.53 & 0 \\
Anesthetist fee & 300.00 & 0 \\
LMA & 6.75 & 0 \\
Autoclaving and processing & 1.25 & 0 \\
Yanchauer suction and tubing & 1.56 & 0 \\
Lubricant & 0.13 & 0 \\
Intravenous catheter & 2.10 & 0 \\
Intravenous tubing & 8.27 & 0 \\
Ringer's lactate, 1 L & 1.23 & 0 \\
Syringes (10×2 cm $\left.{ }^{3}\right)$ & 0.18 & 0 \\
Needles $(18 \mathrm{G}, 25 \mathrm{G})$ & 0.48 & 0 \\
Opsites $\times 2$ & 0.36 & 0 \\
Plastic surgery & & \\
1\% Lidocaine with epinephrine & 0 & 2.08 \\
$(1: 100,000)$ & 75.64 & 22.65 \\
Labor & 59.34 & 11.73 \\
Supplies & $\$ 468.82$ & $\$ 36.46$ \\
Total & & \\
\hline
\end{tabular}

clinic or office setting. In this patient group, $100.0 \%$ have their fasciectomies under local anesthesia with epinephrine, and only $40.0 \%$ have tourniquets. Of those surgeons that operate under local anesthesia, the majority do so for both single digit \pm palmar disease $(100.0 \%)$ and multiple digits \pm palmar disease (100.0\%). Approximately $60.0 \%$ of surgeons in this group also perform fasciectomy for recurrent disease under local anesthesia.

Surgeons reported that tourniquets were used in $67.5 \%$ of cases overall, and local anesthetic with epinephrine was used in $47.3 \%$ of cases. In terms of the spectrum of cases, plastic surgeons operated on patients with both single and multi-digit disease commonly $(46.4 \%$ and $44.5 \%$, respectively) and rarely on recurrent disease (12.0\%). A minority of surgeons refer patients to OT/PT postoperatively $(37.5 \%)$ and use postoperative splints $(45.8 \%)$. Of those that splint, $50.0 \%(8 / 16)$ use night splints and $50 \%$ use immediate postop splints. Night splints are worn for an average of 4 months (range 3-6 months). Immediate postoperative splints are most commonly worn for 2 weeks (range 1-3 weeks).

\section{Discussion}

The Wide-Awake Approach to hand surgery incorporates the use of local anesthetic with lidocaine and epinephrine (one in 100,000 or less) for hemostasis without the use of tourniquet, sedation, or general anesthesia $[11,12,20]$. Our goal in this study was to compare the postoperative outcomes of Dupuytren's patients having surgery with the Wide-Awake Approach vs. with general anesthesia and a tourniquet. We found that complication rates, changes in extension, and individual digital total active motion were not significantly different between the two groups; however, the combined postoperative TAM for all fingers was better in the general anesthesia group rather than the Wide-Awake group. These findings still support the use of the Wide-Awake Approach for Dupuytren's fasciectomy, while simultaneously exemplifying some of the benefits and limitations of WideAwake surgery.

The TAM values in our study are comparable with those in Denkler's analysis of 60 digital fasciectomies done under local anesthesia with epinephrine, where the average MP joint increase in extension was $30.2^{\circ}$, and average increase in PIP joint extension was $22.5^{\circ}$ [12]. Total active motion data and change in extension values are also comparable with that in the literature, for both primary and recurrent disease $[12,27,30,32]$. One possible explanation for the slightly lower TAM values in our Wide-Awake group is that there were four patients in the Wide-Awake group who had surgery for recurrent disease, where there was only one patient in the general anesthetic group who had surgery for recurrent disease (see Table 1).

Complication rates were not significantly different in our two groups in terms of wound infections, digital nerve lacerations, and delayed healing. Denkler [12] noted that his rate of digital nerve transection was $2.4 \%$, similar to others [8]. We would have preferred to report a lower nerve injury rate, but four of our total of 148 fingers suffered nerve lacerations (Table 1). The higher incidence in the general anesthetic group was not statistically significant. The increased blood in the field of the Wide-Awake patients did not appear to increase the incidence of nerve laceration.

General anesthesia complication rates such as nausea and vomiting are well known to occur in up to $1 / 3$ of patients getting this form of treatment [7]. We did not determine the rate of postoperative nausea and vomiting (PONV), the most common side effect of general anesthesia, in our general anesthetic group. The literature estimates the incidence of PONV at $20-40 \%$, which can elongate hospital stays and increase costs $[6,14,15,28,35]$. Obviously, patients undergoing the Wide-Awake Approach with pure local anesthesia only did not suffer any PONV at all, nor did they have to be admitted for general anesthesia related problems.

Although we did not formally evaluate patient comfort levels performed with the Wide-Awake Approach, it is the impression of most of the surgeons who use this technique regularly that the patients suffer no pain after the initial needle poke of the local injection when it is injected slowly, 
when the needle never gets ahead of the wheal of the local anesthesia, when generous volumes are used, and when the local anesthesia is given time to become intense. In addition, patients who feel stressed coming into the new surgical environment usually adapt quite rapidly when they realize there is total numbness, and it becomes for most like a trip to the dentist.

The incidence of Dupuytren's disease has been reported as high as $25 \%$ in Anglo-Saxon males, with much lower rates in Asian and African ethnic groups [1, 10, 17, 25]. This incidence in all groups has shown to increase with age, and a high percentage of patients opt to undergo surgery for the condition. A recent study from Britain determined an incidence of 32.5/100,000/year based on referrals with 20.5/100,000/year opting for surgical fasciectomy [9]. This amounted to 12,000 cases in the UK per year [13]. A second study from France estimated the total cost of $4,179,998$ Euros, for a total of 14,860 hospitalizations for Dupuytren's surgery in 2001 [24]. This represents a huge economic burden, which in Canada is superimposed on a strained public healthcare system facing increasing costs, insufficient capacity, and an aging population with increasing needs.

The cost of performing Dupuytren's surgery in the clinic under local anesthesia in the ambulatory setting is much less expensive than in the main operating room (\$36.46 vs. $\$ 468.82$, a saving of $\$ 432.36$ per patient when compared with fasciectomy under GA). The true cost savings is in all likelihood greater because our study did not take into account medications used for the treatment of postoperative nausea and vomiting following GA or any costs associated unwanted hospital admission due to complications of general anesthesia. Unplanned overnight hospital admissions following Dupuytren's fasciectomy have been reported to occur in $14 \%$ of patients [23]. When expanded into the context of a surgeon who performs 20 fasciectomies for primary disease per year under GA, the cost savings becomes $\$ 10,809.00$.

Our survey indicated that there tended to be two main surgeon preferences with regard to where Dupuytren's surgery is performed and how hemostasis is achieved. Over half of the plastic surgeons at our centers $(75.0 \%)$ perform Dupuytren's surgery in the main OR the majority of the time. These surgeons also use tourniquets in the majority (74.7\%) of cases. General anesthesia is used in only $29.5 \%$ cases, with regional and local anesthesia in the rest. Only $20.8 \%$ of our surgeons use the clinic or office for the majority of their cases. All of these cases are performed under pure local anesthesia with epinephrine (Wide-Awake Approach).

The previously held belief that finger epinephrine injection is contraindicated is no longer valid $[2-4,11$, 12, 18-20, 31, 33, 34, 36]. Thomson et al. showed that the myth of epinephrine-induced digital necrosis was actually most likely generated by procaine [34]. Injection of $1 \mathrm{mg}$ of phentolamine in $1 \mathrm{~cm}^{3}$ of saline reliably reverses epinephrine vasoconstriction in the finger in $1.5 \mathrm{~h}$, whereas it normally takes epinephrine vasoconstriction $6.5 \mathrm{~h}$ to wear off by itself [26].

Some of the limitations of this study include: (1) more multiple digits procedures were performed under general anesthesia than under local anesthesia; (2) it is retrospective; (3) there could be more data on preoperative contracture measurements; (4) some of the costs were difficult to obtain due to the nature of the Canadian healthcare system.

The limitations of Wide-Awake surgery include the following. Wide-Awake hand surgery is challenging because it is not bloodless surgery. Although most adult hand surgery can be performed with the Wide-Awake Approach, Dupuytren's fasciectomy is one of the more difficult operations to perform with this approach because of the bleeding generated by cord dissection adjacent to the digital arteries. Even in our experienced hands, most of us prefer to perform redo Dupuytren's surgery under general anesthesia with a tourniquet. Our study results suggest that better total active motion may be achieved by performing complex multidigit fasciectomy under general rather than local anesthesia. It may be that this enables the surgeon to perform more extensive dissection and excision of diseased fascia with optimal visibility using a tourniquet. It may be wiser for the surgeon who is not familiar with the WideAwake Approach to start with more simple procedures such as carpal tunnel release, trigger finger, etc. However, many Dupuytren's patients are older with comorbidities. If they have only had lidocaine and epinephrine for anesthesia, they simply get up and go home just like when they have been to a dentist. This avoids problems such as nausea and vomiting or unplanned admissions, which are associated with general anesthesia.

Disclosure This study has received no external funding and has no commercial or private sponsors. The authors have no conflicts of interest to disclose.

\section{References}

1. Al-Qattan M. Factors in the pathogenesis of Dupuytren's contracture. J Hand Surg. 2006;31A:1527-34.

2. Altinyazar HC, Ozdemir H, Koca R, Hosnuter M, Demirel CB, Gundogdu S. Epinephrine in digital block: color Doppler flow imaging. Dermatol Surg. 2004;30:508-11.

3. Andrades PR, Olguin FA, Calderon W. Digital blocks with or without epinephrine. Plast Reconstr Surg. 2003;111(5):176970.

4. Bezuhly M, Sparkes GL, Higgins A, Neumeister MW, Lalonde DH. Immediate thumb extension following extensor indicis 
proprius-to-extensor pollicis longus tendon transfer using the wide-awake approach. Plast Reconstr Surg. 2007;119 (5):1507-12.

5. Briedis J. Dupuytren's contracture: lack of complications with the open palm technique. Br J Plast Surg. 1974;27:218-9.

6. Bruns K. Postoperative care and review of complications. Int Anesthesiol Clin. 1982;20(1):27-34.

7. Buck DW, Mustoe TA, Kim JYS. Postoperative nausea and vomiting in plastic surgery. Semin Plast Surg. 2006;20:249-55.

8. Bulstrode NW, Jemec B, Smith PJ. The complications of Dupuytren's contracture surgery. J Hand Surg (Am). 2005;30A:1021-5.

9. Burke FD, Dias JJ, Lunn PG, Bradley M. Providing care for hand disorders: trauma and elective. The Derby Hand Unit experience (1989-1990). J Hand Surg [Br]. 1991;16(1):13-8.

10. Burke FD, Proud G, Lawson IJ, McGeoch KL, Miles JNV. An assessment of the effects of exposure to vibration, smoking, alcohol and diabetes on the prevalence of dupuytren's disease in 97, 537 miners. J Hand Surg (Eur). 2007;32E(4):400-6.

11. Denkler K. A comprehensive review of epinephrine in the finger: to do or not to do. Plast Reconstr Surg. 2001;108:4.

12. Denkler K. Dupuytren's fasciectomies in 60 consecutive digits using lidocaine with epinephrine and no tourniquet. Plast Reconstr Surg. 2005;115:802-10.

13. Dias JJ, Braybrooke J. Dupuytren's contracture: an audit of outcomes of surgery. J Hand Surg (Br). 2006;31B(5):514-21.

14. Fortney JT, Gan TJ, Graczyk S, Wetchler B, Melson T, Khalil S. A Comparison of the efficacy, safety, and patient satisfaction of ondansetron versus droperidol as antiemetics for elective outpatient surgical procedures. Anesth Analg. 1998;86:731-8.

15. Gan TJ, Ginsberg B, Grant AP, Glass PS. Double-blind, randomized comparison of ondansetron and intraoperative propofol to prevent postoperative nausea and vomiting. Anesthesiology. 1996;85:103642.

16. Hamilton WK, Sokoll MD. Tourniquet paralysis. JAMA. 1967;199(1):37.

17. Hueston JT. Dupuytren's contracture. In: Flynn JE, editor. Hand surgery. 3rd ed. Baltimore: Williams and Wilkins; 1982. pp. 797-823.

18. Katis PG. Epinephrine in digital blocks: refuting the dogma. CJEM. 2003;5(4):245-6.

19. Krunic AL, Wang LC, Soltani K, Weitzul S, Taylor RS. Digital anesthesia with epinephrine: an old myth revisited. J Am Acad Dermatol. 2004;51:755-9.

20. Lalonde DL, Bell M, Benoit P, Sparkes G, Denkler K, Chang P. A multicenter prospective study or 3,110 consecutive cases of elective epinephrine use in the fingers and hand: the Dalhousie project clinical phase. J Hand Surg. 2005;30A:1061-7.

21. Leblanc MR, Lalonde J, Lalonde DH. A detailed cost and efficiency analysis of performing carpal tunnel surgery in the main operating room versus the ambulatory setting in Canada. Hand. 2007;2(4):173-8.

22. Macario A, Chang PC, Stempel DB, Brock-Utne JG. A cost analysis of the laryngeal mask airway for elective surgery in adult outpatients. Anesthesiology. 1995;83(2):250-7.

23. Mandal A, Imran D, McKinnel T, Rao T. Unplanned admissions following ambulatory plastic surgery - a retrospective study. Ann R Coll Surg Engl. 2005;87(6):466-8.

24. Maravic M, Landais P. Dupuytren's disease in France-1831 to 2001 - from description to economic burden. J Hand Surg (Br). 2005;30B(5):484-7.

25. Mitra A, Goldstein RV. Dupuytren's contracture in the black population: a review. Ann Plast Surg. 1994;32:619-22.

26. Nodwell T, Lalonde DH. How long does it take phentolamine to reverse adrenaline induced vasoconstriction in the finger and hand? A prospective randomized blinded study: the Dalhousie Project experimental phase. Can J Plast Surg. 2003;11(4):187-9.

27. Orlando JC, Smith JW, Goulian D. Dupuytren's contracture: a review of 100 patients. Br J Plast Surg. 1974;27:211.

28. Palazzo MGA, Strunin L. Anesthesia and emesis. I. Etiology. Can Anaesth Soc J. 1984;31:178-87.

29. Ramamurthy S, Hickey R. Anesthesia. In: Green DP, Hotchkiss RN, Pedersen WC, editors. Green's operative hand surgery. 4th ed. New York: Churchill Livingstone; 1999. p. 22-47.

30. Roush TF, Stern PJ. Results following surgery for recurrent Dupuytren's disease. J Hand Surg. 2000;25A:291-6.

31. Sellens C, Morrison L. Accidental injection of epinephrine by a child: a unique approach to treatment. CJEM. 1999;1(1):34-6.

32. Shaw DL, Wise DI, Holms W. Dupuytren's disease treated by palmar fasciectomy and an open palm technique. J Hand Surg (Br). 1996;21B(4):484-5.

33. Syladis P, Logan A. Digital blocks with epinephrine: an old dogma refuted. J Hand Surg. 1998;23B(I):17-9.

34. Thompson CJ, Lalonde DH, Denkler KA, Feicht AJ. A critical look at the evidence for and against elective epinephrine use in the finger. Plast Reconstr Surg. 2007;119:260-6.

35. Wetchler BV. Anesthesia for ambulatory surgery. 2nd ed. Philadelphia: Lippincott; 1991. p. 463.

36. Wilhelmi BJ, Blackwell SJ, Miller JH, Mancoll JS, Dardano T, Tran A, et al. Do not use epinephrine in digital blocks: myth or truth? Plast Reconstr Surg. 2001;107:393-7. 